\title{
Unterstellte Leseschaften
}

\section{Berit Glanz Memes als Wertungen von Literatur in den sozialen Medien}


„Literatur sollte ... Literatur darf nicht ... Literatur muss ... vervollständige mithilfe deiner Handytastatur" ${ }^{1}$ Autocomplete ${ }^{2}$ ist ein gutes Beispiel dafür, wie technologische Innovationen sehr rasch von den jeweilige Nutzer*innen auf eine kreative und initial nicht in den Funktionen der jeweiligen Innovation angelegten Weise verwendet werden. Entwickelt, um bei der Texteingabe in elektronische Geräte Zeit zu sparen, sind Spiele mit der algorithmisch basierten Autovervollständigungsfunktion mittlerweile ein in den sozialen Medien häufig aufzufindendes Phänomen. Satzanfänge werden mit Hilfe der Funktion ergänzt und können so zu überraschenden, witzigen oder absurden Resultaten führen.

Da die automatische Vervollständigung des Getippten sich an zuvor Formuliertes anlehnt, häufige Wortverwendungen und Kontexte der individuellen User*innen auswertet, bekommt die Funktion etwas Orakelhaftes, wodurch sich vielleicht die Beliebtheit der Anwendung erklärt. ${ }^{3}$ Typisch für das Zusammenspiel von Mensch und Algorithmus ist auch bei der kreativen Nutzung der Autovervollständigungsfunktion die kuratierende Einwirkung des Menschen, der entscheidet, welche Texte veröffentlicht werden, welchen Weg durch die Textvorschläge er nimmt.

Fabian Navarro bezieht das Spiel mit der Autovervollständigungsfunktion in seinem eingangs zitierten, am 9. September 2020 verfassten Tweet auf den Literaturbegriff selbst: Die große Bandbreite der Antworten zeigt einerseits, wie stark sich diese Funktion auf die einzelnen Anwendenden einstellt, und verweist andererseits in den ausgewählten Beispielen der jeweiligen Nutzer*innen auch implizit auf deren Vorstellungen von Literatur:

„Literatur sollte man nicht kuscheln. Literatur darf nicht zu hoch angesetzt werden. Literatur muss leider nicht mehr so lange in Erinnerung bleiben. “4

\footnotetext{
${ }^{1}$ https://twitter.com/FabsNavarro/status/1307310582262005761 (20.1.2021)

${ }^{2}$ Autocomplete, die Autovervollständigkeitsfunktion, findet sich bereits sehr früh in unterschiedlichen digitalen Umfeldern. Sie kann auf Wortebene stattfinden, Sätze vervollständigen oder häufige Suchanfragen voraussagen. Die Autovervollständigung passt sich dabei im Verlauf des Gebrauchs an die Gewohnheiten der jeweiligen Nutzenden an.

${ }^{3}$ Chloe Bryan sieht in ihrem Artikel in dieser Aussagekraft über die Einzelperson die Begründung für die Beliebtheit von Autovervollständigungsmemes und Online-Quizzes. Chloe Bryan: „Predictive text memes: The rush of a personality quiz with none of the work“. In: Mashable, 8.5.2019. https://mashable.com/article/predictive-text-autocomplete-memes

${ }^{4}$ https://twitter.com/fionanongrata/status/1307375026237308930 (20.1.2021).
} 
„Literatur sollte eigentlich reichen. Literatur darf nicht so freigiebig mit Besuchen umgehen. Literatur muss man leben. ${ }^{\text {" }}$

„Literatur sollte man sich auf jeden Fall mal anschauen / Literatur darf nicht nur in der WG langweilig werden / Literatur muss man sich auch nicht unbedingt anhören und es ist auch nicht das Problem der is the product of the intended only for sale“6

„literatur sollte es nicht geben / literatur darf nicht nur oke werden / literatur muss ich noch mal ein bisschen aufräumen“7

Die von verschiedenen Nutzer*innen ausgewählten automatisierten Vervollständigungen lassen exemplarisch eine Enthierarchisierung von Literatur und ein spielerisches Verständnis von dem vermuten, was Literatur in der Gegenwart ausmacht. Es ist naheliegend, auch das Nachdenken über und die Definition von Literatur im Rahmen memetischer Schreibweisen stattfinden zu lassen, ist doch das Schreiben im Internet selbst davon geprägt. Die Sprachwissenschaftlerin Gretchen McCulloch schreibt treffend dazu: „But one thing we know, if we spend more than a minute discussing internet culture, is that it somehow involves a thing called memes. ${ }^{8}$

Der Begriff Meme wurde von Richard Dawkins bereits in den 1970er Jahren geprägt und von Mike Goodwin in einem Wired-Beitrag von 1994 erstmalig auf den Internetdiskurs bezogen. Von Limor Shifman wird der Begriff Meme folgenderweise definiert:

„Statt das Mem als eine einzelne kulturelle Einheit zu beschreiben, die sich erfolgreich fortgepflanzt hat, plädiere ich dafür, ein Internetmem zu definieren als (a) eine Gruppe digitaler Einheiten, die gemeinsame Eigenschaften im Inhalt, in der Form und/oder der Haltung aufweisen; (b) die in bewusster Auseinandersetzung mit anderen Memen erzeugt und (c) von vielen Nutzern über das Internet verbreitet, imitiert und/oder transformiert wurden. Diese Definition ist hilfreich, um Internetmeme als gesellschaftlich konstruierte öffentliche Diskurse $\mathrm{zu}$ analysieren, in denen

${ }^{5}$ https://twitter.com/FeeMinistin/status/1307314886440112128 (20.1.2021).

${ }^{6}$ https://twitter.com/kutter_karl/status/1307383578439942144 (20.1.2021).

${ }^{7}$ https://twitter.com/webersjule/status/1307358209179607041 (20.1.2021).

${ }^{8}$ Gretchen McCulloch: Because Internet: Understanding the New Rules of Language. New York 2019, S. 238. 
verschiedene memetische Varianten unterschiedliche Stimmen und Perspektiven repräsentieren." ${ }^{9}$

Wird Meme also als Begriff für Internetphänomene verwendet, dann beschreibt er einen Vorgang, bei dem Inhalte von großen Usermengen spielerisch aufgegriffen, verändert und aufeinander bezogen werden. Memes fordern dafür eine kreative Tätigkeit der Beteiligten. Sie sind stark intertextuell, verweisen auf komplexe Kommunikationen und sind auch deswegen gut dazu geeignet, zur Ausbildung von Online-Gruppenidentitäten beizutragen. Limor Shifman nennt dies eine „hypermemetische Logik“, da sich Memes rasch und weit ausbreiten und deswegen eine „Von vielen geteilte Umgangssprache, die zahlreiche Bereiche digitaler und nichtdigitaler Ausdrucksformen durchzieht", bilden. ${ }^{10}$

Auch das Gespräch über Literatur in den sozialen Medien ist von memetischen Schreibweisen geprägt, wie der Tweet von Fabian Navarro zu Beginn dieses Textes illustrieren sollte. Auf den verschiedenen Online-Plattformen bilden sich unterschiedliche Leser*innengruppen, die eigene Wertungskriterien für Literatur aushandeln und in diesem Prozess immer wieder auch auf Memes zurückgreifen.

Diese Aushandlungsprozesse selbst sind jedoch keine internetspezifische Neuigkeit, denn die Diskussion, welche Allgemeingültigkeit eine ästhetische Wertung, das heißt ein Geschmacksurteil, überhaupt beanspruchen kann, begleitet als philosophische Grundfrage die Auseinandersetzung mit Kunst im weitesten Sinne. In einer sozial ausgesprochen komplex differenzierten Gesellschaft muss es auf die Frage nach den Kriterien guter Literatur zwangsläufig eine große Vielfalt an Antworten geben. Die Diskussion über die Bewertung von Literatur wird noch zusätzlich erschwert, wenn etablierte Gatekeeper-Institutionen des literarischen Feldes ihren diskursiven Einfluss verlieren, wenn wertende Begriffe wie beispielsweise der des Kitsches ihre herabsetzende Kraft verloren haben und nicht nur die Gruppe der Lesenden, sondern auch die Orte, an denen sie diskutieren, sich immer weiter ausdifferenzieren. Etablierte Orte des Sprechens über Literatur und der Aushandlung von

${ }^{9}$ Limor Shifman: Meme: Kunst, Kultur und Politik im digitalen Zeitalter. Berlin 2014, Position 117.

${ }^{10}$ Shifman: Meme, Position 289. 
Wertungskriterien, wie etwa die Feuilletons der großen Zeitschriften, verlieren an Relevanz, wenn sich beispielsweise in den sozialen Medien Stilgemeinschaften finden, die ganz eigene, neue oder andere Wertungskriterien an Literatur anlegen.

Diese Vervielfältigung der Orte, an denen über literarische Texte diskutiert wird, hat sich durch das Internet noch einmal verstärkt. Das weite Feld des Sprechens über Literatur, des Geschmacksabgleiches, der Diskussion über gute und schlechte Formen literarischen Schreibens ist nicht mehr nur auf analoge Räume beschränkt, sondern verschiebt sich zunehmend in den virtuellen Raum. Laut der ARD/ZDFOnlinestudie von 2019 verbringt die Bevölkerung jeden Tag durchschnittlich 87 Minuten im Internet. ${ }^{11}$

Wenn man untersuchen möchte, wie die Wertung von Literatur und die Aushandlung von Wertungskriterien online abläuft, dann lohnt es sich, einen Blick auf die Literaturdiskurse in den sozialen Medien zu werfen, denn immerhin $21 \%$ der Deutschen nutzen Facebook täglich, gefolgt von Instagram mit $13 \%$. Twitter hingegen wird nur von $2 \%$ der Deutschen täglich verwendet.

Trotz der eigentlich geringen Nutzer*innenzahlen ist die Resonanz einiger sozialer Medien in den etablierten Massenmedien erstaunlich hoch und damit auch die Auswirkung von in den sozialen Medien stattfindenden Literaturdebatten auf das weitere literarische Feld. Besonders für Twitter trifft diese Beobachtung zu, denn die Ablehnung/Abwertung von Twitter als Ort literarischen Diskurses ist ein mittlerweile fest etablierter Topos der Kulturkritik im klassischen Feuilleton, obwohl die Zahl der Nutzenden im deutschsprachigen Raum eigentlich nicht nennenswert ist. Diese über das soziale Medium hinausweisende Reichweite hängt wahrscheinlich damit zusammen, dass auf Twitter viele Medienmenschen, Journalist*innen und Politiker*innen anzutreffen sind, weswegen einzelne Tweets und auf Twitter gesetzte Themen weit über die sozial-medialen Grenzen hinaus strahlen können. ${ }^{12}$

\footnotetext{
${ }^{11}$ http://www.ard-zdf-onlinestudie.de/whatsapponlinecommunities/ (20.2.2021).

12 "Sascha Hölig vom Hans-Bredow-Institut warnt indes vor einer Twitter-Blase. Vor allem in Deutschland seien dort Politiker und Journalisten weitgehend unter sich. ,Das kann zum Problem werden dadurch, dass wir wissen, dass insbesondere Journalisten Twitter als Quelle der Recherche nutzen und als Inspiration dafür, welche Themen interessant sind und wie über sie gesprochen wird', sagt Hölig." https://www.ndr.de/fernsehen/sendungen/zapp/medienpolitik/Wie-Journalisten-mit-Twitter-umgehen,twitter934.html (20.2.2021).
} 
In ihrem Versuch, eine kurze Twitter Literaturgeschichte $\mathrm{zu}$ formulieren, schreiben Elias Kreuzmair und Magdalena Pflock, dass Twitter immer wieder die Frage herausgefordert habe, was wir überhaupt als Literatur anerkennen würden und was unsere Kriterien dafür wären, etwas als Literatur zu bestimmen. ${ }^{13}$ Twitter verändert jedoch nicht nur unser Nachdenken über den Literaturbegriff, sondern bildet auch eine eigene Form der Wertung von Literatur heraus, und dabei spielen Memes eine entscheidende Rolle. Deswegen bleibt ein Nachdenken über mögliche Wertungen von Literatur auf Twitter fruchtlos, wenn es nicht mit einem Nachdenken über die Möglichkeiten memetischer Literaturkritik kombiniert wird.

In einer Zeit, in der die Buchveröffentlichung nicht mehr nur ein einseitiger Sendeprozess ist, sondern sich Leser*innen in ihren sozial-medialen Botschaften stark auf die von ihnen rezipierten Texte beziehen, den eigenen Leseprozess kritisch mit Statusupdates begleiten und das Gelesene an verschiedenen Online-Orten ausführlich oder nur mit einer Sternenvergabe bewerten, sind auch Memes ein relevanter Bestandteil der Kommunikation geworden.

\section{Die Wiederkehr des genialen Autors als Meme}

Genieästhetische Konzepte prägen das literarische Feld weiterhin stark, in enger Verzahnung mit der kanonischen Wertung einzelner Autor*innen, die vielen Lesenden aus dem Schulunterricht noch ein Begriff sind. Es ist auffällig, wie oft sich Literaturinteressierte auf Twitter gerade an einem implizit angenommenen Kanon abarbeiten. Einige Namen bestimmter Autor*innen haben durch diese sozial-mediale Auseinandersetzung mittlerweile selbst eine Art Meme-Status erreicht.

Dazu gehört beispielsweise Peter Handke, dessen aufgebrachte, im Kontext der Nobelpreisvergabe 2019 in einem Interview des Ö1-Morgenjournals getätigte Aussage „Ich komme von Homer, ich komme von Cervantes, lasst mich in Frieden und

\footnotetext{
${ }^{13}$ "Was sich in diesen Titeln artikuliert, ist eine Frage, die Twitter immer wieder herausgefordert hat: Was erkennen wir als Literatur an? Was sind unserer Kriterien dafür, etwas als Literatur zu bestimmen? Ist, wer ein paar Witze und Sentenzen in 280 Zeichen packt, eine literarische Autor*in? Wer ein Buch beim twitteraffinen Frohmann Verlag veröffentlicht hat? Oder muss es Suhrkamp sein?" Elias Kreuzmair, Magdalena Pflock: „Mehr als Twitteratur - Eine kurze Twitter-Literaturgeschichte." In: 54Books, 24.9.2020. Link: https://www.54books.de/mehr-als-twitteratur-eine-kurze-twitter-literaturgeschichte/ (20.2.2021).
} 
stellt mir nie wieder solche Fragen " ${ }^{14} \mathrm{zu}$ einem Meme wurde, das sich rasch ausbreitete. Zu der Memifizierung gehörten viele Anspielungen auf die mögliche Doppeldeutigkeit des „Ich komme von“, beispielsweise „Klar, Sex ist toll, aber seid ihr schonmal von Tolstoi gekommen?“ von @schnalena ${ }^{15}$, Aufforderungen zu einer Autovervollständigung wie „Gib ,Ich komme von Tolstoi“ ein und lass die Autokorrektur deine Antwort formulieren“"von @robindetje ${ }^{16}$ und zahlreiche Kombinationen der Originalaussage mit Reaktionsbildern, wie beispielsweise von @starcaztle ${ }^{17}$, der das Handke-Zitat mit dem Milk-Edinburgh-Meme ${ }^{18}$ verwendet, das einen Mann zeigt, der einer sich sichtlich unwohl fühlenden Frau in einem Club ins Ohr redet.

Ein anderer Autor, dessen Name besonders oft auftaucht, wenn die gefühlte Enge etablierter anspruchsvoller Literatur thematisiert werden soll, ist Martin Walser. „Mein Lebensgefühl mit 23: Brügge sehen und sterben Mein Lebensgefühl heute: Martin Walser lesen und kotzen \#früherwarmehrlametta"19 schreibt @AndreaKlatt2 und @Lata_mariusz schreibt: „Sich selbst bestrafen, \& darum Martin Walser lesen“. ${ }^{20}$ Immer wieder wird auf „den ewigen Kulturgreis “"21 Martin Walser Bezug genommen, oft mit einem impliziten Verabschieden der Vergangenheit: „Wollen wir Martin Walser nicht endlich begraben?“"22; „Ich kenne keinen einzigen Martin Walser-Leser. Gibt es seine Bücher überhaupt oder ist das ein jahrzehntelanger Presse-Hoax? “23 fragt @mitnichten und in den Antworten wird mehrfach geschrieben, dass man diese Bücher vor Jahrzehnten gelesen hätte ${ }^{24}$. „Werfe die Ehen in Philippsburg jetzt in den Müll, bekommt man nirgendwo noch Geld für“25 schreibt @peterdhintz im Februar 2020 und gibt in der Antwort auf seinen Tweet einen Hinweis auf die Überpräsenz Walsers, die Teil seiner erfolgreichen Wiederkehr als Meme in den sozialen Medien

\footnotetext{
14 "Ich komme von Homer, ich komme von Cervantes, lasst mich in Frieden." In: FAZ 16.10.2019.

${ }^{15} \mathrm{https} / / /$ twitter.com/schnalena/status/1184395826946285568 (20.2.2021).

${ }^{16} \mathrm{https} / / /$ twitter.com/robindetje/status/1184394760418672640 (20.2.2021).

${ }^{17}$ https://twitter.com/starcaztle/status/1184403327112626176 (20.2.2021).

${ }^{18} \mathrm{https} / / /$ knowyourmeme.com/memes/milk-edinburgh (20.2.2021).

${ }^{19} \mathrm{https} / / /$ twitter.com/AndreaKlatt2/status/1252879521188995073 (20.2.2021).

${ }^{20} \mathrm{https} / / /$ twitter.com/Lata_mariusz/status/1307220944415535105 (20.2.2021).

${ }^{21}$ https://twitter.com/silbermund/status/62909313408176128 (20.2.2021).

${ }^{22}$ https://twitter.com/david_1312_/status/1297569606945714176 (20.2.2021).

${ }^{23} \mathrm{https}$ ///twitter.com/mitnichten/status/686686969829654528 (20.2.2021).

${ }^{24}$ https://twitter.com/s_krieger/status/686806257282478080 (20.2.2021).

und https://twitter.com/ilsebodenstein/status/687002234769555456 (20.2.2021).

${ }^{25} \mathrm{https} / / /$ twitter.com/peterdhintz/status/1226152161987497985 (20.2.2021).
} 
sein dürfte: „Auch signierte Walser-Ausgaben praktisch wertlos, der Mann unterschreibt einfach alles. ${ }^{26}$

Martin Walser wird so in vielen Tweets zum Symbol einer Literaturform, von der man sich abgrenzen möchte, ein Gruppenbildungsprozess, mit dem sich neue gruppenimmanente Wertungskriterien auf Twitter spielerisch etablieren.

Darüber hinaus gibt es auch zahlreiche Tweets, die sich dem Autor mit einer betont humorvollen Respektlosigkeit nähern, dabei spielen seine buschigen Augenbrauen eine entscheidende Rolle. „Martin Walser hat heut morgen gut gefrühstückt, sich die Augenbrauen gekämmt, ist nach Frankfurt gefahren und hat ihn umgeboxt, den Ranicki“" ${ }^{27}$ oder „Romane von Martin \#Walser gibt es jetzt als Sonderausgabe mit struppigen Riesen-Augenbrauen, mit denen man beim Lesen laut rumrascheln

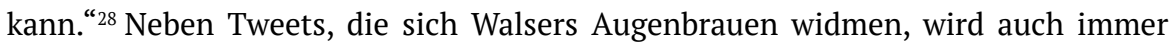
wieder auf den Wohnort Martin Walsers angespielt. Er ist der „Nebelwerfer vom

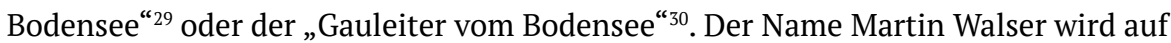
Twitter auch wiederholt als Teil einer Vossianischen Antonomasie verwendet, um damit andere Denker und Literaten prägnant zu beschreiben. Klaas Huizing ist der Martin Walser der Theologie, ${ }^{31}$ Habermas der Martin Walser der Wissenschaft, ${ }^{32}$ Ingo Schulze der Martin Walser des Ostens. ${ }^{33}$

Der Differenzierung von Limor Shifman folgend, die Virals, gründerbasierte und egalitäre Meme voneinander abgrenzt, ist „Martin Walser“ ein egalitäres Meme. Es gibt keine einzelne Version, die sich wie ein Lauffeuer verbreitet, wie bei einem Viral, und die „Martin Walser“-Tweets sind auch keine Ableitungen von einer auslösenden viralen Quelle, wie bei einem gründerbasierten Meme. Stattdessen gibt es viele verschiedene Varianten, die sich aber alle auf das Muster ,Martin Walser als unfreiwillig komischer Repräsentant eines veralteten Systems ‘ beziehen. Diese Logik wird

\footnotetext{
${ }^{26} \mathrm{https}: / /$ twitter.com/peterdhintz/status/1226154208623308803 (20.2.2021).

${ }^{27}$ https://twitter.com/geruchtekellner/status/380359161240104960 (20.2.2021).

${ }^{28} \mathrm{https} / / /$ twitter.com/stporombka/status/303952815800332288 (20.2.2021).

${ }^{29} \mathrm{https}$ ///twitter.com/istuetzle/status/596739210293485568 (20.2.2021).

${ }^{30} \mathrm{https}: / /$ twitter.com/Georgias1/status/287218756789350400 (20.2.2021).

${ }^{31} \mathrm{https}$ ://twitter.com/bellabahr/status/1294939394927984641 (20.2.2021).

${ }^{32}$ https://twitter.com/helenhockay/status/1141223291539075074 (20.2.2021).

${ }^{33} \mathrm{https} / / /$ twitter.com/shorstkotte/status/1298208113904975874 (20.2.2021).
} 
auch bedient, wenn ein Walser Titel, nämlich „Tod eines Kritikers“, mit einem twitterspezifischen Begriff umformuliert wird: „Tod eines Drunterkommentierers ist ein Roman von Martin Walser, der 2002 im Suhrkamp Verlag erschienen ist. “"

Und selbst Tweets, die nur eine eigene Rezeptionserfahrung schildern, tragen indirekt zur Memifizierung des Autorennamens bei. Am 17. Januar 2019 schreibt beispielsweise @JudithCVogt: „Ich musste in der Oberstufe ,Ein fliehendes Pferd“von Walser lesen. Deutschlehrer: ,Wird euch gefallen, bisschen Sex, bisschen Crime.'Es ging um sexuell frustrierte Leute im Alter unseres Deutschlehrers. Ich denke bis heute mit Schaudern daran. “35 Das unangenehme und oft durch Institutionen wie Schule oder Universität erzwungene Lesen von Walsers Büchern wird auf Twitter immer wieder thematisiert, genauso wie die Unlust, ein weiteres Buch von ihm anzufangen. ${ }^{36}$ Die memetische Häufigkeit, mit der Martin Walser in Gesprächen zur Literatur auf Twitter erscheint, thematisiert @BenschMoritz: „Was würdet ihr alle nur machen, wenn es MW nicht gäbe?"37

Hier zeigt sich das anfangs bereits erwähnte, interessante Phänomen des Literaturdiskurses auf Twitter: Es wird sehr viel Zeit darauf verwendet, sich von einem literarischen Kanon abzugrenzen, neue Kriterien zu definieren und sich gegenseitig zu versichern, dass man tatsächlich die Freiheit habe, sich von etablierten Wertungskriterien abzuwenden: „niemand muss walser lesen“ schreibt @derloeffelbein.. ${ }^{38}$

\section{Dekanonisierung als Spiel und Parodie}

Spielerische Dekanonisierung ist eine gute Zusammenfassung einer bestimmten Form von Literaturdiskurs auf Twitter, die sich beispielsweise in Umfragen „Welcher dieser Autoren ist der überschätzteste ${ }^{\text {“39 }}$, in Vorschlägen zum Auf- und Abstieg einzelner Autor*innen in einem imaginierten Literatur-DAX ${ }^{40}$ oder Fragen wie: „Wenn

\footnotetext{
${ }^{34}$ https://twitter.com/cssr_aw/status/833702076135911425 (20.2.2021).

${ }^{35}$ https://twitter.com/JudithCVogt/status/1085997891611697153 (20.2.2021).

${ }^{36} \mathrm{https} / / /$ twitter.com/Wolfseule/status/1213019985666281473 (20.2.2021).

${ }^{37} \mathrm{https}: / /$ twitter.com/BenschMoritz/status/1081635181302370309 (20.2.2021).

${ }^{38} \mathrm{https://twitter.com/derloeffelbein/status/1273197331656200192} \mathrm{(20.2.2021).}$

${ }^{39} \mathrm{https}: / /$ twitter.com/Johannes42/status/1052211479473246208 (20.2.2021).

${ }^{40} \mathrm{https}: / /$ twitter.com/BurnRHopemaster/status/1296858498110836736 (20.2.2021).
} 
du einen Schriftsteller opfern müsstest, um Corona zu beenden, welcher wäre es und warum Hermann Hesse?" ${ }^{41}$ äußert. Das Dekanonisierungsspiel erstreckt sich nicht nur auf konkrete Autor*innen, sondern auch auf literarische Texte, die aufgrund ihrer Omnipräsenz als Klischee wahrgenommen werden. Am 22. September, dem kalendarischen Herbstanfang, schreibt Johanna Dürrholz auf Twitter „Der Erste, der jetzt Rilke zitiert, wird gecancelt." 42

Kanon ist politisch und das Bewusstsein dafür ist auf Twitter sehr groß. Auch deswegen sind Wertungsfragen und Kommentare zur Literatur in Tweets oft entweder mit Forderungen nach einer politischen Ästhetik oder Überlegungen zur strukturellen Diskriminierung im literarischen Feld verknüpft: „Die vier Typen der Literatur: -Männer um die 17, die ihren Penis entdecken -Männer um die 30, die vom Leben enttäuscht sind -Männer um die 50, die ihren Penis suchen -Männer um die 80, die aufs Meer starren“43 schreibt @sei_riots in einem Tweet. Dass derartige kritischspöttische Tweets zu populären Ästhetiken und im Literaturbetrieb hochdekorierten Autor*innen und Texten auf Twitter Konjunktur haben, dürfte an der für die sozialen Medien typischen Öffnung und Enthierarchisierung der Kommunikationsräume liegen. Dirk von Gehlen schreibt:

„Auch wenn über die Aufmerksamkeitsmaschinen von sozialen Medien wie Facebook, Twitter, Instagram oder auch von Messengern wie WhatsApp, die diese Form der horizontalen Kommunikation weiter beschleunigt haben, häufig vor allem aus einer problematisierenden Perspektive gesprochen wird, lohnt es sich festzuhalten: Die Demokratisierung der Publikationsmittel hat in erster Linie denen eine Stimme gegeben, die sich im 20. Jahrhundert nie (und die in den Jahrhunderten zuvor erst recht nicht) auf diese Weise äußern konnten." ${ }^{44}$

Diese Politisierung von Literatur erstreckt sich nicht nur auf Autor*innen, sondern auch auf bestimmte Schreibweisen und -ästhetiken. Besonders klischeebeladene und unfreiwillig komische Beispiele von männlichen Autoren, die weibliche Figuren

${ }^{41} \mathrm{https} / / /$ twitter.com/mlewandowsky/status/1281694443129708551 (20.2.2021).

${ }^{42}$ https://twitter.com/Duerrholz/status/1308496279257972736 (20.2.2021).

${ }^{43} \mathrm{https} / / /$ twitter.com/sei_riots/status/986984310992498688 (20.2.2021).

${ }^{44}$ Dirk van Gehlen: Meme. Digitale Bildkulturen. Berlin 2020, S. 18. 
schreiben, oder von weißen Autor*innen, die POC-Figuren schildern, werden in verschiedenen Subreddits - Unterforen des Social-News-Aggregators Reddit gesammelt, darunter beispielsweise das 2017 gegründete r/menwritingwomen mit 385.000 Mitgliedern oder das 2019 gegründete r/whitepeoplewritingPOC mit 5600 Mitgliedern. Es gibt ein wachsendes kritisches Bewusstsein für politische Probleme und ethische Implikationen literarischen Schreibens.

Tweets und Videos, in denen solche als problematisch empfundenen Schreibästhetiken parodiert werden - oftmals Schreibweisen, die sich ignorant gegenüber den Realitäten strukturell Diskriminierter zeigen - erreichen auf Twitter regelmäßig viralen Status ${ }^{45}$ und es entstehen darauf aufbauende Memes, wie beispielsweise Tweets, in denen Männerfiguren ebenso klischeebeladen beschrieben werden wie Frauenfiguren. Eine Form dieses Umkehr-Memes ist das Meme \#dichterdran, bei dem Autoren überspitzt so beschrieben wurden, wie es oft bei Autorinnen der Fall ist und war. ${ }^{46}$

Bei dieser Form einer Literaturkritik durch Parodie, indem spöttisch als negativ und ignorant abgewertete Schreibweisen nachgeahmt werden, bleiben die Wertungskriterien implizit. Durch Retweets und Favs werden diese in der Parodie implizit geäußerten Wertungen dann bestätigt und beeinflussen den Literaturbegriff der sich konstituierenden Fav-Gemeinschaft.

\section{Ein Buch muss die Axt sein für das Wesentliche, was man nur mit dem Herzen gut sieht - Memifizierung einzelner literarischer Texte}

Nachdem nun bereits die memetische Wirkung einzelner Autor*innen und Memes, die aus der kritischen Parodie von Schreibweisen entstehen, beschrieben wurden, will ich zum Ende noch einen Ausblick auf eine Sorte Memes geben, die für die Literaturwissenschaft einige spannende Fragen aufwirft: Warum werden literarische Texte bzw. Auszüge aus literarischen Texten zur Meme-Vorlage?

\footnotetext{
${ }^{45}$ https://twitter.com/lasersushi/status/1304707938548932609 (20.2.2021).

${ }^{46} \mathrm{https}: / /$ twitter.com/tiffstevenson/status/1301944631719059457 (20.2.2021).
} 
Der Bot @gregorsamsbot ${ }^{47}$ tweetet beispielsweise seit Juni 2018 regelmäßig den Textanfang von Franz Kafkas Erzählung „Die Verwandlung“ und kombiniert ihn mit passenden Versatzstücken aus anderen literarischen Texten: „Als Gregor Samsa eines Morgens aus unruhigen Träumen erwachte, fand er die zwei Flügel des Greifen und nahm sie in großer Freude mit sich nach Hause“48 oder „Als Gregor Samsa eines Morgens aus unruhigen Träumen erwachte, fand er nahezu alle Plätze besetzt " ${ }^{49}$. Der Bot spielt dabei mit der memetischen Verwendung von Kafkas erstem Satz, der als Ssnowclone zum Meme geworden ist. Ein snowclone ist ein zur Vorlage gewordenes Klischee oder ein formelhaft verwendeter Satz, der Begriff wurde 2004 als Ausdruck für formelhaft klischierte Sprachmuster formuliert und wird seitdem besonders für spezifische Memes verwendet. ${ }^{50}$ Doch Gregor Samsa taucht nicht nur im deutschsprachigen Raum als Ssnowclone auf, bei dem der Satzanfang mit verschiedenen Halbsätzen beendet wird, sondern ist in Einzeltweets und in Bot-Form auch auf Englisch anzufinden. Der @GregorSamsaBot tweetet seit Januar 2016 regelmäßig neue Variationen des Satzes.

Es gibt zahlreiche andere Beispiele von literarischen Zitaten, die auf diese Weise zu Ssnowclones geworden sind und auf Twitter zirkulieren, von Franz Kafkas „Axt für das gefrorene Meer in uns“ bis zu Antoine de Saint-Exupérys beliebtem Zitat aus dem Kleinen Prinzen „Man sieht nur mit dem Herzen gut ...“.

Neben einzelnen Satzzitaten aus literarischen Texten werden auch Gedichte auf Twitter aufgegriffen und als Formatvorlage immer wieder innovativ verändert. Im Kontext der „Avenidas“-Debatte ${ }^{51}$ zirkulierten zahlreiche Abwandlungen des Originals von 1953 als Meme auf Twitter. Das Gedicht eignet sich aufgrund

\footnotetext{
${ }^{47} \mathrm{Im}$ Folgenden werden die Programmierer*innen der Bots in einer Fußnote angegeben, wenn sie sich in der Bio des Twitterbots zu erkennen geben. Der @gregorsamsbot wurde laut Twitterbio von @greg00r und @lavievagabone erstellt (16.2.2021).

${ }^{48} \mathrm{https} / / /$ twitter.com/gregorsamsbot/status/1309475512268599300 (20.2.2021).

${ }^{49} \mathrm{https} / / /$ twitter.com/gregorsamsbot/status/1308569540700315655 (20.2.2021).

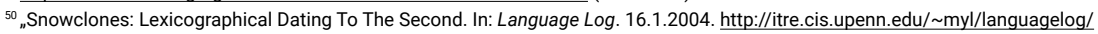
archives/000350.html (16.2.2021).

${ }^{51}$ Im Herbst 2017 entbrannte eine Debatte um das Gedicht „Avenidas“, das zu diesem Zeitpunkt noch auf der Fassade der Alice Salomon Hochschule in Berlin angebracht war. Studierende der Hochschule hatten einen Antrag auf Entfernung des Gedichtes gestellt, da sie das Gedicht als Reproduzierung von Sexismus deuteten. Die Debatte wurde in den folgenden Monaten an vielen Orten medial aufgegriffen. Das Referat Hochschulkommunikation der Alice Salomon Hochschule hat das mediale Ereignis in einem ausführlichen Pressespiegel abgebildet: https://www.ash-berlin.eu/hochschule/organisation/referat-hochschulkommunikation/pressespiegel-fassadendebatte/ $(16.2 .2021)$.
} 
seines sehr schlichten Formats nicht nur perfekt als Vorlage für spielerische Aneignungen, sondern auch als Basis für einen Bot, den im Januar 2018 veröffentlichten @gomringador. ${ }^{52}$

Der @ sosweetbot ${ }^{53}$ retweetet seit Februar 2018 Tweets, die sprachlich Bezug auf das Gedicht „This is just to say“ (1938) von William Carlos Williams nehmen. Das Gedicht war jedoch bereits zuvor im Herbst 2017 Quelle eines erfolgreichen Memes, bei dem Inhalte und Sprachfetzen des Gedichtes mit populären Songtexten vermischt wurden. ${ }^{54}$ Beispielsweise in Verbindung mit Lou Bega: „a little bit of cold plums in my life a little bit of icebox by my side a little bit of breakfast is what you need a little bit of forgiveness, what I seek a little bit of delicious, thats those plums a little bit of sweet plums all night long a little bit of cold plums here I am “ ${ }^{55}$, in Verbindung mit Carly Rae Jepsen: „HEY i just met you and this is CRAzy but the plums in the icebox $i$ ate them maybe “ ${ }^{56}$ und in Verbindung mit den Beatles „Blackbird singing in the dead of night Take these breakfast plums and learn to eat All your life You were only waiting for a meal this cold and sweet ". ${ }^{7}$

Der User @Zorknogg tweetet am 29. November 2017: „William Carlos Williams: ,hey what happened to that poem i wrote way back about the plums and icebox?' Ppl in 2017: ,it’s a huge meme now “" ${ }^{58}$ Warum und wie Gedichte zur Vorlage für Memes werden, ist bis jetzt kaum näher untersucht worden. Vermutlich hat es einerseits mit bestimmten leicht nachahmbaren sprachlichen Qualitäten des Ausgangstextes zu tun, man kann konkrete Poesie einfacher kopieren als Barocksonette, andererseits müssen die Texte bei einer hinreichenden Anzahl von Menschen als Vorlage abrufbar sein, um überhaupt als Meme zu funktionieren. Das geschieht besonders dann, wenn ein Gedicht Debattengegenstand ist, wie bei „Avenidas“, oder aktiver Teil des schulliterarischen Kanons, wie bei „This is to say.“

\footnotetext{
${ }^{52}$ In der Twitterbio des Bots wird auf einen Twitteraustausch verwiesen, in dem Kathrin Passig als Programmiererin des Bots erkennbar wird. (16.2.2021)

${ }^{53}$ Die Twitterbio des Bots verweist auf @mellymeldubs und @johnrladd als Urheber*innen des Bots (16.2.2021).

${ }^{54} \mathrm{https}: / /$ www.nme.com/news/plum-icebox-meme-william-carlos-williams-poem-1934-2166850 (20.2.2021).

${ }^{55}$ https://twitter.com/thwphipps/status/935465303202557952 (20.2.2021).

${ }^{56} \mathrm{https} / / /$ twitter.com/Choplogik/status/935655741054009344 (20.2.2021).

${ }^{57}$ https://twitter.com/theseantcollins/status/935743261041414144 (20.2.2021).

${ }^{58} \mathrm{https}$ ///twitter.com/Zorknogg/status/935671997958361088 (20.2.2021).
} 
Die memifizierte Ästhetik eines literarischen Textes oszilliert immer zwischen Spott und Anerkennung. Sie enthält nicht grundsätzlich eine kritische oder positive Wertung, sondern es muss anhand von Einzelfällen betrachtet werden, wie diese Memes entstehen, sich entwickeln und verbreiten, ob sie kritisch oder affirmativ gelesen werden können. Memes sind hochdynamisch, Semantiken und formale Verfahren ändern sich konstant und ihre Untersuchung ist bis jetzt kaum systematisiert. Es lässt sich jedoch eindeutig feststellen, dass in den sozialen Medien neue Formen der Wertung von Literatur und neue Kanonisierungspraktiken entstehen und Memes in diesem Prozess eine entscheidende Rolle spielen. 




\section{Duisburg-Essen Publications online}

offen im Denken

$\mathbf{U b} \mid \begin{gathered}\text { universitäts } \\ \text { bibliothek }\end{gathered}$

In: Unterstellte Leseschaften. Kulturwissenschaftliches Institut Essen, 29. bis 30. September 2020

Dieser Text wird via DuEPublico, dem Dokumenten- und Publikationsserver der Universität Duisburg-Essen, zur Verfügung gestellt. Die hier veröffentlichte Version der E-Publikation kann von einer eventuell ebenfalls veröffentlichten Verlagsversion abweichen.

DOI: $\quad 10.37189 /$ duepublico/74183

URN: urn:nbn:de:hbz:464-20210503-132239-2

Dieses Werk kann unter einer Creative Commons

Namensnennung -Nicht kommerziell - Keine Bearbeitungen 4.0 Lizenz (CC BY-NC-ND 4.0) genutzt werden. 